\title{
Chaotic Electron Trajectories in a Planar Wiggler Free-Electron Laser
}

\author{
A. Soleyman and H. Mehdian* \\ Department of Physics, Teacher Training University, Tehran, Iran
}

(Received July 26, 2006)

\begin{abstract}
The motion of an electron in a planar wiggler with an axial guide field is found to be nonintegrable. When taking into account the effects of self-fields of the beam, it is confirmed that the motion of an electron in a planar wiggler with a guide field may be chaotic. There is evidence of chaos from numerical calculations of nonzero Lyapunov exponents using different approaches of Benettin's method which are described and compared. Very accurate Poincaré maps are also performed.
\end{abstract}

PACS numbers: 41.60.Cr

\section{Introduction}

The chaotic electron trajectories in a planar wiggler are investigated in the presence of a guild field [1]. It is seen that the trajectories of electron are chaotic for both neglecting and considering the self-fields. Nonintegrability of the electron motion is demonstrated in this work by calculating nonzero Lyapunov exponents. These are derived by using different approaches of Benettin's [2] method which are described in detail. The largest Lyapunov exponent [3] is estimated conditions and also by using the tangent vector method. The good agreement between the different results makes us confident that, in some circumstances, a nonzero Lyapunov exponent exists. Poincaré maps $[4,5]$ are also generated very accurately, to confirm that some trajectories are chaotic. The results have been compared to the work done by using a helical wiggler $[6,7]$.

\section{Theoretical formulation of the problem}

The motion of one electron in a free-electron laser (FEL) with a planar wiggler $\boldsymbol{B}_{\omega}$ and a guide field $\boldsymbol{B}_{0}$ is considered. The self-fields produced by the

\footnotetext{
*corresponding author; e-mail: Mehdian@saba.tmu.ac.ir
} 
electron beam are neglected. The motion of the electron takes place in the following magnetic field:

$$
\boldsymbol{B}=\boldsymbol{B}_{0}+\boldsymbol{B}_{\omega}=B_{0} \hat{\boldsymbol{e}}_{\boldsymbol{z}}+B_{\omega} \sin \left(k_{\omega} z\right) \hat{\boldsymbol{e}}_{\boldsymbol{y}} .
$$

The vector potential $\boldsymbol{A}$ can be defined by

$$
\begin{aligned}
& \boldsymbol{A}=\boldsymbol{A}_{\omega}+\boldsymbol{A}_{0}, \quad \boldsymbol{A}_{\omega}=-\frac{B_{\omega}}{k_{\omega}} \cos \left(k_{\omega} z\right) \hat{\boldsymbol{e}}_{\boldsymbol{x}}, \quad \boldsymbol{A}_{0}=B_{0} x \hat{\boldsymbol{e}}_{\boldsymbol{y}} \\
& \boldsymbol{A}=-\frac{B_{\omega}}{k_{\omega}} \cos \left(k_{\omega} z\right) \hat{\boldsymbol{e}}_{\boldsymbol{x}}+B_{0} x \hat{\boldsymbol{e}}_{\boldsymbol{y}} .
\end{aligned}
$$

The corresponding Hamiltonian is

$$
H=\gamma m c^{2}=\sqrt{1+\frac{p^{2}}{m^{2} c^{2}}} m c^{2}=\sqrt{m^{2} c^{4}+p^{2} c^{2}} .
$$

Making the use of canonical momentum

$$
P_{i}=p_{i}-\frac{e}{c} A_{i},
$$

therefore, we have

$$
p_{x}=P_{x}-\frac{e B_{\omega}}{c k_{\omega}} \cos \left(k_{\omega} z\right), \quad p_{y}=P_{x}+\frac{e B_{0}}{c} x, \quad p_{z}=P_{z} .
$$

Substituting Eqs. (5) into Eq. (3), it yields

$$
H=\sqrt{m^{2} c^{4}+c^{2}\left[\left(P_{x}-\frac{e B_{\omega}}{c k_{\omega}} \cos \left(k_{\omega} z\right)\right)^{2}+\left(P_{y}+\frac{e B_{0}}{c} x\right)^{2}+P_{z}^{2}\right]} .
$$

The Hamiltonian Eq. (3) can be rewrite as canonical momentum

$$
\frac{H}{m c^{2}}=\sqrt{1+\left[\left(\frac{P_{x}}{m c}-\frac{e B_{\omega}}{m c^{2} k_{\omega}} \cos \left(k_{\omega} z\right)\right)^{2}+\left(\frac{P_{y}}{m c}+\frac{e B_{0}}{m c^{2}} x\right)^{2}+\frac{P_{z}^{2}}{m^{2} c^{2}}\right]} .
$$

Since the Hamiltonian is not an explicit function of time, $H$ is a constant of motion. For numerical calculation, dimensionless variables are defined as follows:

$$
\begin{aligned}
& \hat{P}_{i} \equiv \frac{P_{i}}{m c}, \quad \hat{x} \equiv k_{\omega} x, \quad \hat{z} \equiv k_{\omega} z, \quad \gamma \equiv \frac{H}{m c^{2}}, \quad \tau \equiv c k_{\omega} t, \quad a_{\omega} \equiv \frac{e B_{\omega}}{m k_{\omega} c^{2}}, \\
& \Omega_{c} \equiv \frac{e B_{0}}{m c}, \quad \hat{\Omega}_{c} \equiv \frac{\Omega_{c}}{c k_{\omega}} \quad \text { with } \quad \Omega_{c}=\frac{e B_{0}}{m c} .
\end{aligned}
$$

Using these definitions, the Hamiltonian equation can be written as

$$
\gamma=\hat{H}=\sqrt{1+\left(\hat{P}_{x}-a_{\omega} \cos \hat{z}\right)^{2}+\left(\hat{P}_{y}+\hat{\Omega}_{c} \hat{x}\right)^{2}+\hat{P}_{z}^{2}} .
$$

Making the use of $\dot{q}_{k}=\partial H / \partial p_{k}, \dot{p}_{k}=-\partial H / \partial q_{k}$, we have

$$
\begin{aligned}
& \frac{\mathrm{d} \hat{x}}{\mathrm{~d} \tau}=\frac{1}{\gamma}\left(\hat{P}_{x}-a_{\omega} \cos \hat{z}\right)=\frac{\partial \hat{H}}{\partial \hat{P}_{x}}, \quad \frac{\mathrm{d} \hat{y}}{\mathrm{~d} \tau}=\frac{1}{\gamma}\left(\hat{P}_{y}+\hat{\Omega}_{c} \hat{x}\right)=\frac{\partial \hat{H}}{\partial \hat{P}_{y}}, \\
& \frac{\mathrm{d} \hat{z}}{\mathrm{~d} \tau}=\frac{1}{\gamma} \hat{P}_{z}=\frac{\partial \hat{H}}{\partial \hat{P}_{z}}, \quad \frac{\mathrm{d} \hat{P}_{x}}{\mathrm{~d} \tau}=-\frac{1}{\gamma}\left(\hat{\Omega}_{c} \hat{P}_{y}+\hat{\Omega}_{c}^{2} \hat{x}\right)=-\frac{\partial \hat{H}}{\partial \hat{x}},
\end{aligned}
$$




$$
\frac{\mathrm{d} \hat{P}_{y}}{\mathrm{~d} \tau}=0=-\frac{\partial \hat{H}}{\partial \hat{y}}, \quad \frac{\mathrm{d} \hat{P}_{z}}{\mathrm{~d} \tau}=-\frac{1}{\gamma}\left(a_{\omega} \hat{P}_{x} \sin \hat{z}-a_{\omega}^{2} \cos \hat{z} \sin \hat{z}\right)=-\frac{\partial \hat{H}}{\partial \hat{z}} .
$$

We have plotted the trajectory of an electron in the $(\hat{x}, \hat{y})$ plane. The motion looks chaotic for some initial conditions as seen in Fig. 1.

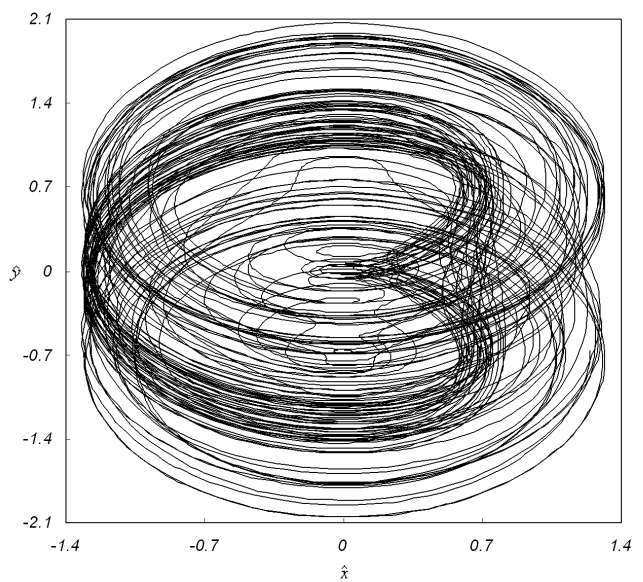

Fig. 1. Projection of an electron trajectory on the $(\hat{x}, \hat{y})$ plane, for $B_{0}=1.98 \mathrm{~T}$, $B_{\omega}=1.5 \mathrm{~T}, \hat{\Omega}_{c}=2, \gamma=3$, and for the following initial conditions, $\hat{x}=0.17, \hat{y}=0$, $\hat{z}=0, \hat{P}_{x}=0, \hat{P}_{y}=0, \hat{P}_{z}=2.13$.

The chaotic trajectories of electron can be confirmed by performing Poincaré section and calculating nonzero Lyapunov exponents.

For using Poincaré mapping, in addition to the obtained Hamiltonian, we need to find the constants of motion by employing the canonical transformation.

By integrating equation of motion Eq. (8) through the fourth-order RungeKutta method, the Hamiltonian becomes

$$
H=\sqrt{m^{2} c^{4}+c^{2}\left[\left(P_{x}-\frac{e B_{\omega}}{c k_{\omega}} \cos \left(k_{\omega} z\right)\right)^{2}+\left(P_{y}+\frac{e B_{0}}{c} x\right)^{2}+P_{z}^{2}\right]} .
$$

The generating function may be defined as

$$
F_{2}\left(x, y, z, P_{1}, P_{2}, P_{3}\right)=\left(P_{1}-\frac{e B_{0}}{c} y\right) x+P_{2}\left(y-\frac{P_{1} c}{e B_{0}}\right)+P_{3} z,
$$

where $F_{2}$ is independent of time and

$$
P_{i}=\frac{\partial F_{2}}{\partial q_{i}}, \quad Q_{i}=\frac{\partial F_{2}}{\partial P_{i}}, \quad k=H+\frac{\partial F_{2}}{\partial t} .
$$

Substituting $x, y, z, P_{x}, P_{y}, P_{z}$ in terms of new coordinates, we have

$$
H=\sqrt{m^{2} c^{4}+c^{2}\left[\left(\frac{e B_{0}}{c} Q_{2}+\frac{e B_{\omega}}{c k_{\omega}} \cos \left(k_{\omega} Q_{3}\right)\right)^{2}+P_{2}^{2}+P_{3}^{2}\right]} .
$$


The Hamiltonian is not an explicit function of time; $H$ is a constant of motion. As expected, $H$ is independent of the parameters $Q_{1}$ and $P_{1}$.

Having failed in finding a third constant of motion, Poincaré maps have been plotted to demonstrate the nonintegrability of the motion. For this purpose, the following normalized equations of motion, derived from Eq. (11), have been solved numerically

$$
\begin{aligned}
\frac{\mathrm{d} \hat{Q}_{1}}{\mathrm{~d} \tau}=0, \quad \frac{\mathrm{d} \hat{Q}_{2}}{\mathrm{~d} \tau}=\frac{\hat{P}_{2}}{\gamma}, \quad \frac{\mathrm{d} \hat{Q}_{3}}{\mathrm{~d} \tau}=\frac{\hat{P}_{3}}{\gamma}, \\
\frac{\mathrm{d} \hat{P}_{1}}{\mathrm{~d} \tau}=0, \quad \frac{\mathrm{d} \hat{P}_{2}}{\mathrm{~d} \tau}=-\frac{1}{\gamma}\left(\hat{\Omega}_{c}^{2} \hat{Q}_{2}+\hat{\Omega}_{c} a_{\omega} \cos \left(\hat{Q}_{3}\right)\right), \\
\frac{\mathrm{d} \hat{P}_{3}}{\mathrm{~d} \tau}=\frac{1}{\gamma}\left(a_{\omega} \hat{\Omega}_{c} \sin \left(\hat{Q}_{3}\right) \hat{Q}_{2}+a_{\omega}^{2} \sin \left(\hat{Q}_{3}\right) \cos \left(\hat{Q}_{3}\right)\right) .
\end{aligned}
$$

Here the dimensionless variables $\hat{Q}_{i}$ and $\hat{P}_{i}$ are defined as $\hat{Q}_{i} \equiv k_{\omega} Q_{i}, \hat{P}_{i} \equiv P_{i} / m c$ $(i=1,2,3)$.

The motion occurs in a three-dimensional space $\left(\hat{Q}_{2}, \hat{Q}_{3}, \hat{P}_{2}\right)$. The plane $\left(\hat{Q}_{2}, \hat{P}_{2}\right)$ with $\hat{Q}_{3}=0(\bmod 2 \pi)$ is chosen to be the Poincaré surface of section. The numerical method used is a fourth-order Runge-Kutta one. The following figures (Figs. 2, 3, 4) show nonintegrable surface-of-section plots for different magnetic fields.

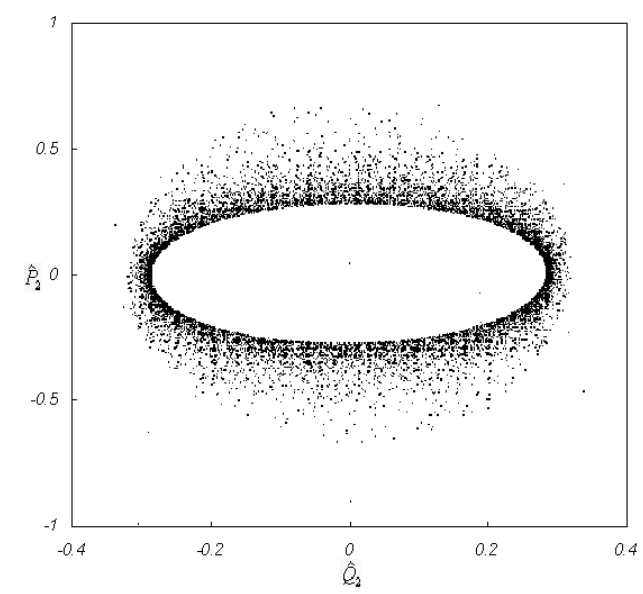

Fig. 2. Surface-of-section plots for chaotic trajectories with $\hat{Q}_{3}=0(\bmod 2 \pi)$, for $\gamma=3, B_{0}=5.45 \mathrm{~T}, B_{\omega}=1.5 \mathrm{~T}$, and $\hat{\Omega}_{c}=5.5$.

As it is seen in Fig. 5 trajectories motion of electron is chaotic. The existence of chaotic trajectories is confirmed by calculating nonzero Lyapunov exponents by two approaches. The first consists in considering two nearby trajectories with an initial tangential vector of the norm $d_{0}$. The distance $d_{n}$ between those trajectories is calculated numerically, and as it is seen $d_{n} / d_{0}$ is greater than a quantity of 2.7 , or for every fixed time interval $\Delta \tau=30 \mathrm{~s}$, we renormalize $d_{n}$ to $d_{0}$. 


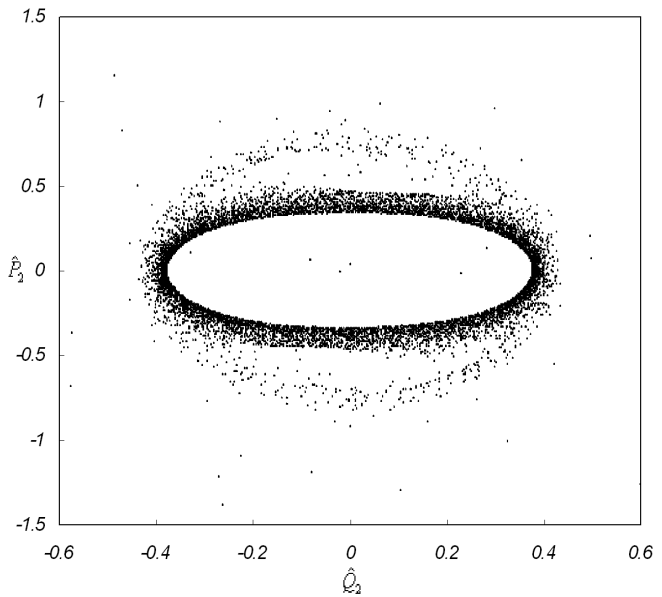

Fig. 3. Surface-of-section plots for chaotic trajectories with $\hat{Q}_{3}=0(\bmod 2 \pi)$, for $\gamma=3, B_{0}=4.21 \mathrm{~T}, B_{\omega}=1.5 \mathrm{~T}$, and $\hat{\Omega}_{c}=4.25$.

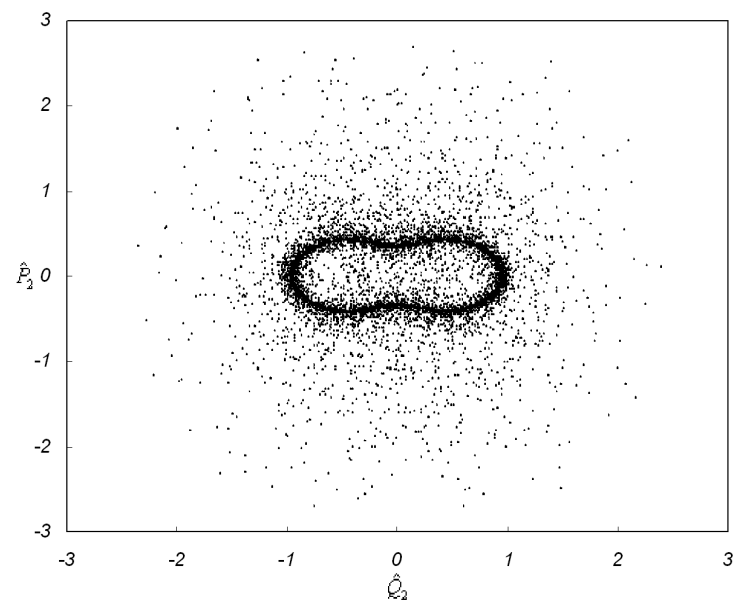

Fig. 4. Surface-of-section plots for chaotic trajectories with $\hat{Q}_{3}=0(\bmod 2 \pi)$, for $\gamma=3, B_{0}=2.97 \mathrm{~T}, B_{\omega}=1.5 \mathrm{~T}$, and $\hat{\Omega}_{c}=3$.

The Lyapunov exponent corresponding to the Poincaré map shown in Fig. 5 is given by

$$
\sigma=\lim _{t_{n_{\max }} \rightarrow \infty, d_{0} \rightarrow 0} \frac{1}{t_{n_{\max }}} \sum_{1}^{n_{\max }} \ln \left(\frac{d_{n}}{d_{0}}\right) .
$$

The value of $\theta$ versus time is given in Fig. 6a.

The second approach consists in integrating the differential equation on the tangent vector $[2,8] \omega_{i}=\left(x_{1 i}-x_{2 i}\right)$, where $x_{1 i}$ and $x_{2 i}$ are the coordinates of two neighboring trajectories at the same time $t$. The time evolution for $W$ is found by linearizing 


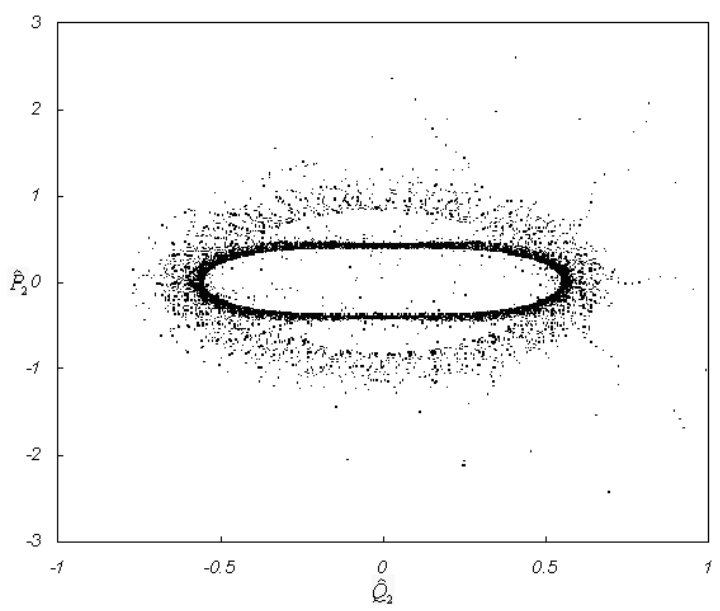

Fig. 5. Nonintegrable surface-of-section plots with $\hat{Q}_{3}=0(\bmod 2 \pi)$, for $\gamma=3$, $B_{0}=1.73 \mathrm{~T}, B_{\omega}=1.5 \mathrm{~T}$, and $\hat{\Omega}_{c}=1.75$.
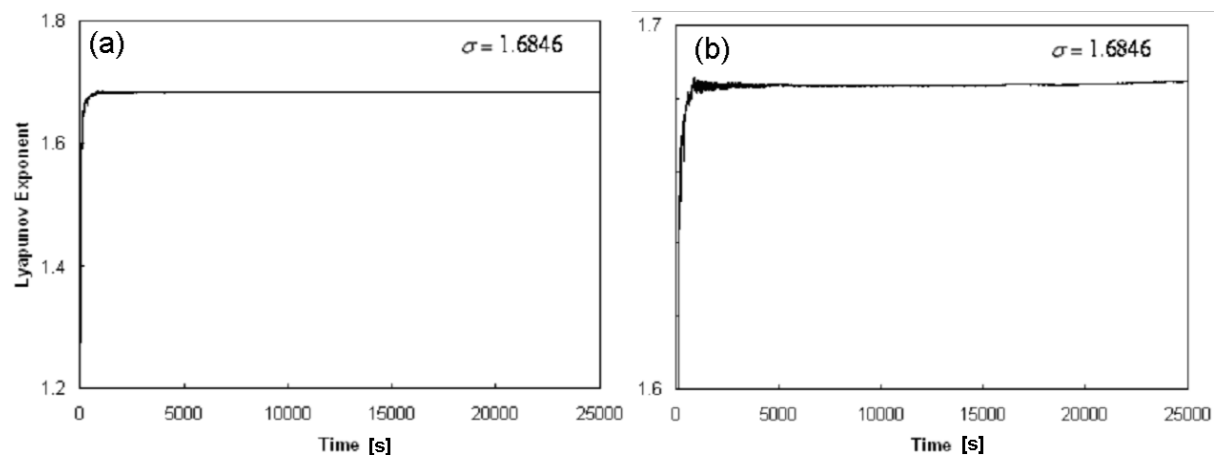

Fig. 6. Lyapunov exponents corresponding to the trajectory of Fig. 5 obtained by (a) numerically integrating two trajectories and (b) with the linearized equations: one corresponding to Fig. 5 with the following initial conditions: $\hat{Q}_{2}=\hat{Q}_{3}=0, \hat{P}_{2}=$ $3.7 \times 10^{-2}, \hat{P}_{3}=2.36$, and another one with very close initial conditions: $\hat{Q}_{2}^{\prime}=\hat{Q}_{2}$, $\hat{Q}_{3}^{\prime}=\hat{Q}_{3}, \hat{P}_{2}^{\prime}=\hat{P}_{2}, \hat{P}_{3}^{\prime}=\hat{P}_{3}\left(1+10^{-6}\right)$.

$$
\frac{\mathrm{d} x_{i}}{\mathrm{~d} t}=V_{i}(X)
$$

to obtain

$$
\frac{\mathrm{d} W}{\mathrm{~d} t}=M[X(t)] W
$$

where

$$
M=\frac{\partial V}{\partial X} .
$$

Here $d(t)=\|W(t)\|$ is derived by integrating Eq. (9) numerically. The corresponding Lyapunov exponent is given by 


$$
\sigma=\lim _{t \rightarrow \infty} \frac{1}{t}[\log d(t)-\log d(0)]
$$

Regarding the Poincaré mapping (Fig. 5) and Lyapunov exponent (Eq. (16)), we conclude that the trajectory motion of electron in a planar wiggler is chaotic even without considering self-fields.

The value of $\sigma$ versus time is given in Fig. 6b.

\section{The effects of self-fields in chaotic trajectories}

To investigate the effects of self-fields let us consider the motion of relativistic electron in a uniform axial field $\boldsymbol{B}_{0} \hat{\boldsymbol{e}}_{\boldsymbol{z}}$, the planar wiggler $\boldsymbol{B}_{\omega}=B_{\omega} \sin \left(k_{\omega} z\right) \hat{\boldsymbol{e}}_{\boldsymbol{y}}$ and self-electric and self-magnetic fields produced by a relativistic non-neutral electron beam. The beam is assumed to have a constant average axial velocity $V_{\mathrm{b}} \hat{\boldsymbol{e}}_{\boldsymbol{z}}$ and of radius $r_{\mathrm{b}}$ [4]. The beam has a uniform density profile as

$$
n_{\mathrm{b}}^{0}= \begin{cases}n_{\mathrm{b}}=\text { const }, & 0 \leq r \leq r_{\mathrm{b}} \\ 0, & r \geq r_{\mathrm{b}}\end{cases}
$$

The self-fields $\boldsymbol{E}_{\mathrm{s}}$ and $\boldsymbol{B}_{\mathrm{S}}$ and the scalar and vector potentials, $\Phi_{\mathrm{s}}$ and $\boldsymbol{A}_{\mathrm{s}}$ have been obtained as

$$
\begin{aligned}
& \boldsymbol{E}_{\mathrm{s}}=-\frac{m \omega_{\mathrm{pb}}^{2}}{2 e}\left(x \hat{\boldsymbol{e}}_{\boldsymbol{x}}+y \hat{\boldsymbol{e}}_{\boldsymbol{y}}\right), \\
& \boldsymbol{B}_{\mathrm{s}}=\frac{m \omega_{\mathrm{pb}}^{2} \beta_{\mathrm{b}}}{2 e c}\left(y \hat{\boldsymbol{e}}_{\boldsymbol{x}}-x \hat{\boldsymbol{e}}_{\boldsymbol{y}}\right), \\
& \Phi_{\mathrm{s}}=\frac{m \omega_{\mathrm{pb}}^{2}}{4 e}\left(x^{2}+y^{2}\right)=\frac{m \omega_{\mathrm{pb}}^{2}}{4 e} r^{2}, \\
& \boldsymbol{A}_{\mathrm{s}}=\frac{m \omega_{\mathrm{pb}}^{2}}{4 e} \beta_{\mathrm{b}} r^{2} \hat{\boldsymbol{e}}_{\boldsymbol{z}}=\beta_{\mathrm{b}} \Phi_{\mathrm{s}} \hat{\boldsymbol{e}}_{\boldsymbol{z}},
\end{aligned}
$$

where $\beta_{\mathrm{b}}=V_{\mathrm{c}} / c, \omega_{\mathrm{pb}}=\left(4 \pi e^{2} n_{\mathrm{b}} / m\right)^{1 / 2}, \omega_{\mathrm{pb}}$ and $n_{\mathrm{b}}$ are plasma frequency and density of beam, respectively.

Considering the self-fields effects the Hamiltonian (Eq. (3)) becomes

$$
H=\sqrt{m^{2} c^{4}+(\boldsymbol{P} c+e \boldsymbol{A})^{2}-e \Phi_{\mathrm{s}}}=\gamma m c^{2}-e \Phi_{\mathrm{s}},
$$

where

$$
\begin{aligned}
& \Phi_{\mathrm{s}}=\frac{m \omega_{\mathrm{pb}}^{2}}{4 e}\left(x^{2}+y^{2}\right)=\frac{m \omega_{\mathrm{pb}}^{2}}{4 e} r^{2}, \quad \boldsymbol{A}=B_{0} x \hat{\boldsymbol{e}}_{\boldsymbol{x}}-\frac{B_{\omega}}{k_{\omega}} \cos \left(k_{\omega} z\right) \hat{\boldsymbol{e}}_{\boldsymbol{x}}+\beta_{\mathrm{b}} \Phi_{\mathrm{s}} \hat{\boldsymbol{e}}_{\boldsymbol{z}}, \\
& A_{\omega}=\frac{B_{\omega}}{k_{\omega}}, \quad \boldsymbol{V}_{0}=V_{\omega} \sin \left(k_{\omega} z\right) \hat{\boldsymbol{e}}_{\boldsymbol{y}}+V_{\mathrm{b}} \hat{\boldsymbol{e}}_{\boldsymbol{z}} .
\end{aligned}
$$

Inserting the self-fields effects in the canonical momentums definition, we have

$$
p_{x}=P_{x}-\frac{m \hat{\Omega}_{\omega}}{k_{\omega}} \cos \left(k_{\omega} z\right), \quad p_{y}=P_{y}+m \hat{\Omega}_{0} x, \quad p_{z}=P_{z}+\frac{m \omega_{\mathrm{pb}}^{2}}{4 c} \beta_{\mathrm{b}} r^{2}
$$


where

$$
\hat{\Omega}_{0, \omega}=\frac{e B_{0, \omega}}{m c} .
$$

Therefore, the Hamiltonian of the system by introducing the following dimensionless variables and parameters:

$$
\begin{aligned}
& \hat{P}_{i}=\frac{P_{i}}{m c}, \quad \hat{x}=k_{\omega} x, \quad \hat{y}=k_{\omega} y, \quad \hat{z}=k_{\omega} z, \quad \hat{H}=\frac{H}{m c^{2}}, \quad \tau=c k_{\omega} t, \\
& a_{\omega}=\frac{e B_{\omega}}{m k_{\omega} c^{2}}, \quad \Omega_{c}=\frac{e B_{0}}{m c}, \quad \hat{\Omega}_{c}=\frac{\Omega_{c}}{c k_{\omega}}=\frac{e B_{0}}{m c^{2} k_{\omega}}, \quad \hat{\omega}_{\mathrm{pb}}=\frac{\omega_{\mathrm{pb}}}{c k_{\omega}}, \quad \hat{\Phi}_{\mathrm{s}}=\frac{e \Phi_{\mathrm{s}}}{m c^{2}}
\end{aligned}
$$

may be written as

$$
\begin{aligned}
\hat{H}= & \sqrt{1+\left(\hat{P}_{x}-a_{\omega} \cos \hat{z}\right)^{2}+\left(\hat{P}_{y}+\hat{\Omega}_{c} \hat{x}\right)^{2}+\left(\hat{P}_{z}+\frac{\hat{\omega}_{\mathrm{pb}}^{2}}{4} \beta_{b}\left(\hat{x}^{2}+\hat{y}^{2}\right)\right)^{2}} \\
& -\frac{\hat{\omega}_{\mathrm{pb}}^{2}}{4}\left(\hat{x}^{2}+\hat{y}^{2}\right) .
\end{aligned}
$$

Making the use of $\dot{q}_{k}=\partial H / \partial p_{k}, \dot{p}_{k}=-\partial H / \partial q_{k}$ the Hamilton equations can be written as

$$
\begin{aligned}
& \frac{\mathrm{d} \hat{x}}{\mathrm{~d} \tau}=\frac{1}{\gamma}\left(\hat{P}_{x}-a_{\omega} \cos \hat{z}\right)=\frac{\partial \hat{H}}{\partial \hat{P}_{x}}, \quad \frac{\mathrm{d} \hat{y}}{\mathrm{~d} \tau}=\frac{1}{\gamma}\left(\hat{P}_{y}+\hat{\Omega}_{c} \hat{x}\right)=\frac{\partial \hat{H}}{\partial \hat{P}_{y}} \\
& \frac{\mathrm{d} \hat{z}}{\mathrm{~d} \tau}=\frac{1}{\gamma} \hat{P}_{z}+\frac{\beta_{\mathrm{b}}}{\gamma} \hat{\Phi}_{s}=\frac{\partial \hat{H}}{\partial \hat{P}_{z}}, \quad \frac{\mathrm{d} \hat{P}_{x}}{\mathrm{~d} \tau}=-\hat{\Omega}_{c} \frac{\mathrm{d} \hat{y}}{\mathrm{~d} \tau}-\hat{x} \beta_{\mathrm{b}} \frac{\hat{\omega}_{\mathrm{pb}}^{2}}{2} \frac{\mathrm{d} \hat{z}}{\mathrm{~d} \tau}+\frac{\hat{\omega}_{\mathrm{pb}}^{2}}{2} \hat{x}=-\frac{\partial \hat{H}}{\partial \hat{x}} \\
& \frac{\mathrm{d} \hat{P}_{y}}{\mathrm{~d} \tau}=-\hat{y} \beta_{\mathrm{b}} \frac{\hat{\omega}_{\mathrm{pb}}^{2}}{2} \frac{\mathrm{d} \hat{z}}{\mathrm{~d} \tau}-\frac{\hat{\omega}_{\mathrm{pb}}^{2}}{2} \hat{y}=-\frac{\partial \hat{H}}{\partial \hat{y}}, \quad \frac{\mathrm{d} \hat{P}_{z}}{\mathrm{~d} \tau}=-a_{\omega} \frac{\mathrm{d} \hat{x}}{\mathrm{~d} \tau} \sin \hat{z}=-\frac{\partial \hat{H}}{\partial \hat{z}}
\end{aligned}
$$

The set of equations is solved numerically using a fourth-order Runge-Kutta method. Figure 7 shows the projection of the electron motion on the $(\hat{x}, \hat{y})$ plane in the case of some initial conditions. The motion looks chaotic for high beam densities and becomes more and more regular as the density decreases. At this stage, one can conclude that the chaos seems to be strongly correlated with the density of the beam.

Figure 8 shows two very close electron trajectories when the density of the beam is assumed to be zero, and, again, when $\Omega_{c}$ is smaller than its critical value. Figure 9 , in contrast, shows two trajectories starting from the same initial case $\left(n_{\mathrm{b}}=2 \times 10^{11} \mathrm{~cm}^{-3}\right)$, while all other parameters are the same as in Fig. 8 . A strong divergence between the two trajectories can be observed. The corresponding Lyapunov exponent has been calculated in order to check whether the two trajectories have an exponential rate of divergence. First, the nonzero Lyapanov exponent is calculated using a first approach of Benettin's method.

We consider the two trajectories having an initial tangential vector with the norm $d_{0}$. A sequence of distances $d_{n}$ corresponding to these trajectories is calculated numerically. For every fixed time interval $\Delta \tau$, or for every fixed distance 


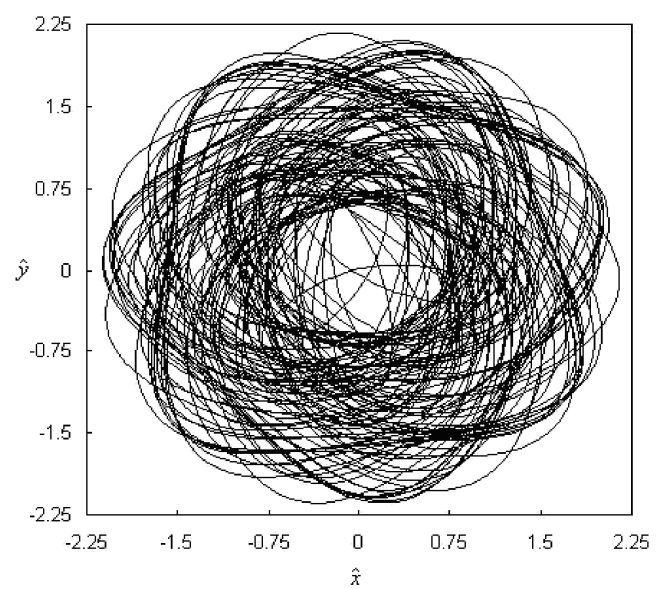

Fig. 7. Projection of an electron trajectory on the $(\hat{x}, \hat{y})$ plane, for $n_{b}=2 \times 10^{11} \mathrm{~cm}^{-3}$, $\beta_{b}=0.91, B_{0}=0.715 \mathrm{~T}, B_{\omega}=715 \mathrm{Gs}, \hat{\Omega}_{c}=2, \hat{H}=3$, and for the following initial conditions: $\hat{x}=-0.25, \hat{y}=-0.93, \hat{z}=0, \hat{P}_{x}=1.85, \hat{P}_{y}=0.5, \hat{P}_{z}=2.36$.

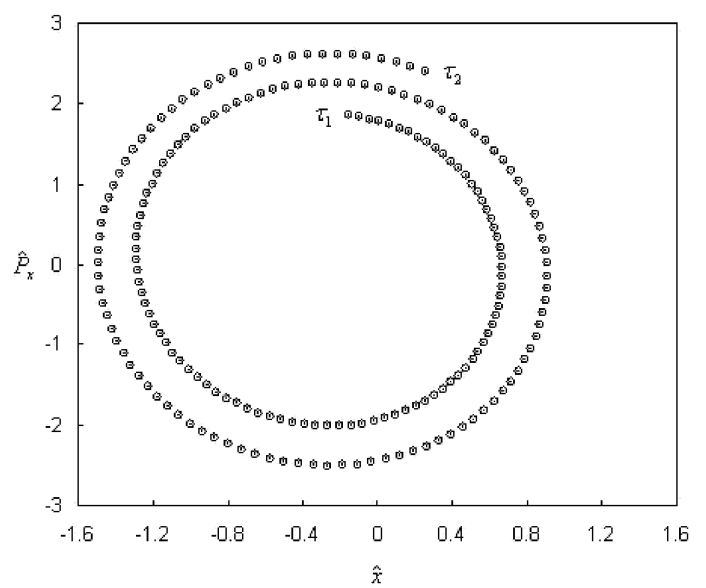

Fig. 8. Projection of two nearby electron trajectories on the $(\hat{x}, \hat{y})$ plane, for $\beta_{b}=0.91$, $B_{0}=0.715 \mathrm{~T}, B_{\omega}=715 \mathrm{Gs}, \hat{\Omega}_{c}=2, \hat{H}=3$, between the two times $\tau_{1}=1245 \mathrm{~s}$ and $\tau_{2}=1295 \mathrm{~s}$, and for the following initial conditions: $\hat{x}_{1}=\hat{x}_{2}=-0.25, \hat{y}_{1}=\hat{y}_{2}=-0.93$, $\hat{z}_{1}=\hat{z}_{2}=0, \hat{P}_{x 1}=\hat{P}_{x 2}=1.85, \hat{P}_{1 y}=0.5, \hat{P}_{2 y}=\hat{P}_{1 y}\left(1+5 \times 10^{-10}\right), \hat{P}_{z 1}=\hat{P}_{z 2}=2.36$.

ratio $d_{n} / d_{0} \approx 2.7$, we renormalized $d_{n}$ to $d_{0}$; that is to say, we consider another trajectory such that the new initial conditions have an initial tangential vector with the norm $d_{0}$.

The Lyapunov exponent is defined as

$$
\sigma \approx \lim _{n \rightarrow \infty, d_{0} \rightarrow 0} \frac{1}{\tau} \sum_{n} \ln \left(\frac{d_{n}}{d_{0}}\right)
$$




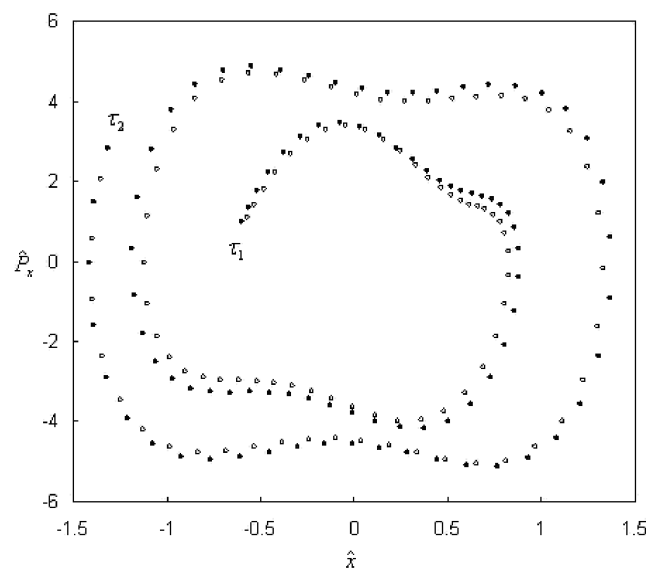

Fig. 9. Projection of two nearby electron trajectories on the $(\hat{x}, \hat{y})$ plane, for $n_{b}=$ $2 \times 10^{11} \mathrm{~cm}^{-3}, \beta_{b}=0.91, B_{0}=0.715 \mathrm{~T}, B_{\omega}=715 \mathrm{Gs}, \hat{\Omega}_{c}=2, \hat{H}=3$, between the two times $\tau_{1}=1245 \mathrm{~s}$ and $\tau_{2}=1295 \mathrm{~s}$, and for the same initial conditions as in Fig. 8.

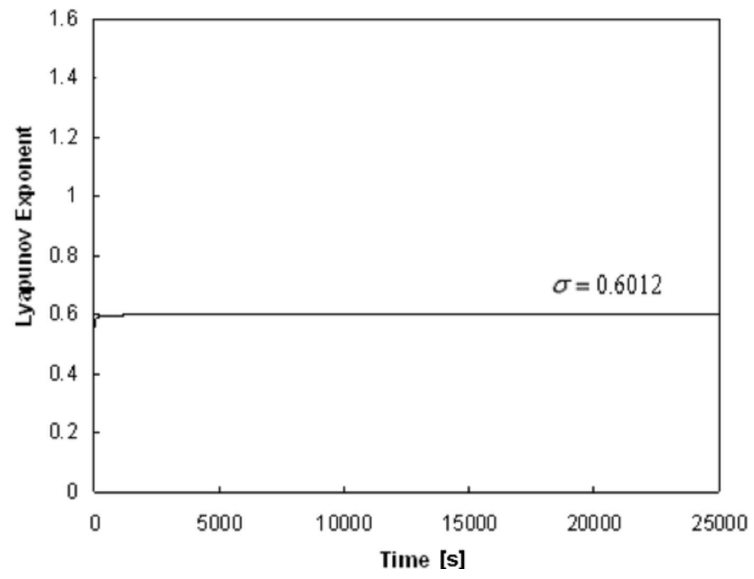

Fig. 10. Lyapunov exponent corresponding to the trajectories shown in Fig. 9, when integrating two trajectories and renormalizing every fixed time $\Delta \tau=30 \mathrm{~s}$ or every fixed distance ratio $d_{n} / d_{0} \approx 2.7$.

and its approximate numerical values, as obtained in our calculations for finite $n$ and $d_{0}$, are shown in Fig. 10. The convergence of our results with respect to $d_{0}$ was verified.

The Lyapunov exponent is also calculated using another approach consisting of integrating the differential equation on the tangent vector $[2] \omega_{i}=\left(x_{i}-x_{j}\right)$, where $x_{j}$ are the coordinates of a point on a trajectory at time $\tau$ defined by the initial condition $X_{0}$, and $x_{i}$ are the coordinates of a point on a nearby trajectory at the same time, $\tau$, determined by the initial condition $X_{0}+\Delta X_{0}$. The time evolution for $W$ is found by linearizing the equation 


$$
\frac{\mathrm{d} x_{i}}{\mathrm{~d} \tau}=V_{i}(X)
$$

to obtain

$$
\frac{\mathrm{d} W}{\mathrm{~d} \tau}=M(X(\tau)) W
$$

where

$$
M=\frac{\partial V}{\partial X} .
$$

By integrating Eq. (23) numerically, we calculate the quantity $d=\|W(\tau)\|$, leading to $d_{n}$ when renormalizations are necessary. Then, the Lyapunov exponent is given by Eq. (25). In most cases, renormalizations are necessary as $d$ becomes too large. They were performed for every fixed time interval or distance ratio $d_{n} / d_{0}$.

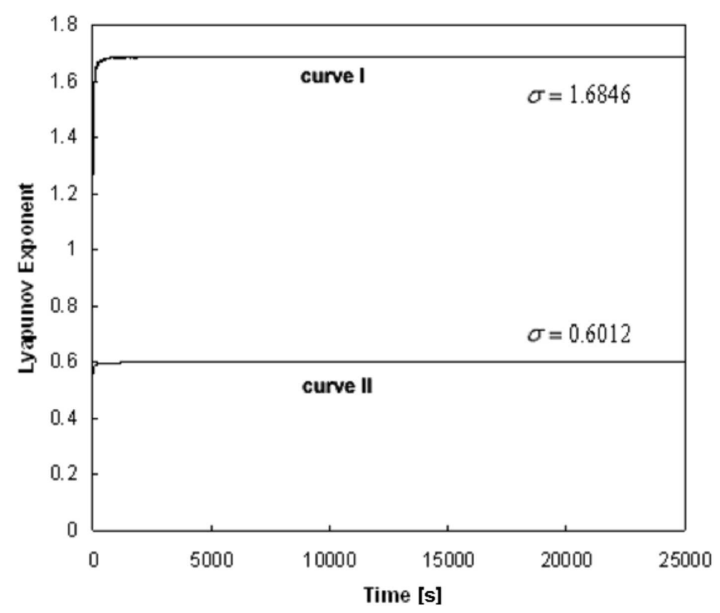

Fig. 11. Lyapunov exponent corresponding to the trajectories shown in Fig. 9 (curve II), and the curve I shows the numerical results obtained for the Lyapunov coefficient when self-fields are neglected.

The curve I in Fig. 11 shows the numerical results obtained for the Lyapunov coefficient when self-fields are neglected. The curve II, in contrast, shows that, when self-fields are considered, the Lyapunov exponent is smaller than in the previous case.

\section{Conclusion}

Using canonical transformation, only two constants of motion have been found. Nevertheless, a guiding center has been determined, which reduces the number of equations of motion. By performing Poincaré sections and calculating Lyapunov exponents, it has been shown that the motion of the one electron in uniform magnetic field and in the field of a planar wiggler can be chaotic. 
Considering the effects of self-fields and using Poincaré mapping and Lyapunov exponent we have obtained that the chaotic trajectory of motion is reduced. Comparing the results of the present work to the ones of the chaotic motion of electron in a helical wiggler derived in [7] we conclude the following points:

a) Although the chaotic behavior is not reported for electron trajectory in a helical wiggler, this behavior is seen in a planar one.

b) Although the self-fields effects cause the chaotic behavior for electron in a helical wiggler, these effects reduce the chaotic behavior for electron in a planar one.

Therefore, we can claim that the self-fields in a planar wiggler has the roles of adding guiding fields for a helical wiggler.

\section{References}

[1] H.P. Freund, J.M. Antonsen, Principle of Free-Electron Lasers, 2nd ed., Chapman and Hall, London 1996.

[2] S.N. Rasband, Chaotic Dynamics of Nonlinear Systems, Wiley, New York 1990; G. Benettin, L. Galgani, J.M. Strelcyn, Phys. Rev. A 14, 2338 (1976).

[3] F. Varosi, T.M. Antonsen Jr., E. Ott, Phys. Fluids A 3, 1017 (1991).

[4] C. Chen, R.C Davidson, Phys. Fluids B 2, 171 (1990).

[5] C. Chen, R.C. Davidson, Phys. Rev. A 43, 5541 (1991).

[6] A. Bourdier, L. Michel-Lours, Phys. Rev. E 49, 3353 (1994).

[7] S. Saviz, H. Mehdian, Acta Phys. Pol. A 107, 895 (2005).

[8] J. Lichtenberg, M.A. Lieberman, Regular and Stochastic Motion, Springer-Verlag, New York 1993. 